

\title{
On special values of standard $L$-functions of Siegel cusp eigenforms of genus 3
}

\author{
par ANH TUAN DO et KIRILl VANKOV
}

\begin{abstract}
RÉSUMÉ. Nous calculons explicitement les valeurs spéciales de la fonction $L$ standard $L\left(s, F_{12}\right.$, St $)$ aux points critiques $s \in\{-8,-6$, $-4,-2,0,1,3,5,7,9\}$, où $F_{12}$ est l'unique forme parabolique (à un scalaire près) de Siegel, de degré 3 et de poids 12 , qui a été construite par Miyawaki. Ces valeurs sont proportionnelles au produit des normes de Petersson du carré symétrique de la fonction $\Delta$ de Ramanujan et de la forme parabolique de poids 20 pour $\mathrm{SL}_{2}(\mathbb{Z})$ avec un facteur rationnel et une certaine puissance de $\pi$. Nous utilisons la méthode de Rankin-Selberg et la projection holomorphe pour calculer ces valeurs. A notre connaissance, c'est le premier exemple d'une fonction $L$ standard d'une forme parabolique de Siegel de degré 3 où les valeurs spéciales peuvent être calculées explicitement.
\end{abstract}

ABSTRACT. We explicitly compute the special values of the standard $L$-function $L\left(s, F_{12}\right.$, St $)$ at the critical points $s \in\{-8,-6$, $-4,-2,0,1,3,5,7,9\}$, where $F_{12}$ is the unique (up to a scalar) Siegel cusp form of degree 3 and weight 12, which was constructed by Miyawaki. These values are proportional to the product of the Petersson norms of symmetric square of Ramanujan's $\Delta$ and the cusp form of weight 20 for $\mathrm{SL}_{2}(\mathbb{Z})$ by a rational number and some power of $\pi$. We use the Rankin-Selberg method and apply the Holomorphic projection to compute these values. To our knowledge this is the first example of a standard $L$-function of Siegel cusp form of degree 3 , when the special values can be computed explicitly.

\section{Introduction}

In the remarkable paper [10] I. Miyawaki considered certain Siegel cusp forms of degree 3 , and on the basis of some numerical calculations, he was able to make interesting conjectures about the degeneration of the standard and spinor $L$-functions associated to such cusp forms. Several years later T. Ikeda [7] proved Miyawaki's conjecture related to the standard

Manuscrit reçu le 2 janvier 2014, révisé le 30 juillet 2014, accepté le 5 septembre 2014.

Mathematics Subject Classification. 11F67, 11F46.

Mots-clefs. special values of $L$-functions, Siegel modular forms, Rankin-Selberg method. 
$L$-function. Basically, he was able to construct an explicit lifting from Siegel cusp forms of degree $r$ to Siegel cusp forms of degree $r+2 n$. In particular, it turns out that the cusp form of degree 3 and weight 12 is a basic example of this lifting (for $r=1, n=1$ ).

Recall that Miyawaki constructed his numerical examples by means of theta functions with spherical functions. Namely, let $E_{8}$ be the unique even unimodular lattice of rank 8 i.e.,

$$
E_{8}=\left\{\begin{array}{l|l}
t \\
\left(x_{1}, \ldots, x_{8}\right) \in \mathbb{R}^{8} & \begin{array}{l}
2 x_{i} \in \mathbb{Z}(i=1, \ldots, 8), \\
x_{1}+\cdots+x_{8} \in 2 \mathbb{Z}, \\
x_{i}-x_{j} \in \mathbb{Z}
\end{array}
\end{array}\right\},
$$

and

$$
Q=\left(\begin{array}{llllllll}
1 & 0 & 0 & i & 0 & 0 & 0 & 0 \\
0 & 1 & 0 & 0 & i & 0 & 0 & 0 \\
0 & 0 & 1 & 0 & 0 & i & 0 & 0
\end{array}\right)
$$

be $3 \times 8$ matrix. Then the theta series

$$
\begin{aligned}
F_{12}(Z) & \left.=\sum_{v_{1}, v_{2}, v_{3} \in E_{8}} \Re\left(\operatorname{det}\left(Q \cdot\left(v_{1}, v_{2}, v_{3}\right)\right)^{8}\right) \exp \left(\pi i \sigma\left(\left\langle v_{i}, v_{j}\right\rangle\right) Z\right)\right) \\
& =\sum_{N>0} a(N) \exp (2 \pi i \sigma(N Z))
\end{aligned}
$$

is a cusp form of weight 12 with respect to $\operatorname{Sp}_{3}(\mathbb{Z})$, where $\left(\left\langle v_{i}, v_{j}\right\rangle\right)$ denotes the matrix composed by the entries, which are the scalar product of two vectors $v_{i}$ and $v_{j}, Z \in \mathfrak{H}^{3}, \sigma$ is the trace operator, and

$$
\begin{aligned}
\mathrm{Sp}_{n}(\mathbb{Z}) & =\left\{M \in \operatorname{Mat}_{2 n}(\mathbb{Z}) \mid M\left(\begin{array}{cc}
0 & \mathrm{I}_{n} \\
-\mathrm{I}_{n} & 0
\end{array}\right)^{t} M=\left(\begin{array}{cc}
0 & \mathrm{I}_{n} \\
-\mathrm{I}_{n} & 0
\end{array}\right)\right\}, \\
\mathfrak{H}^{3} & =\left\{Z={ }^{t} Z=X+i Y \mid X, Y \in \operatorname{Mat}_{3}(\mathbb{R}), Y>0\right\} .
\end{aligned}
$$

Therefore, the explicit Fourier expansion can be computed

$$
\begin{gathered}
F_{12}=1 \cdot q^{\left(\begin{array}{lll}
1 & \frac{1}{2} & \frac{1}{2} \\
\frac{1}{2} & 1 & \frac{1}{2} \\
\frac{1}{2} & \frac{1}{2} & 1
\end{array}\right)}+164 \cdot q^{\left(\begin{array}{ccc}
1 & 0 & 0 \\
0 & 1 & 0 \\
0 & 0 & 1
\end{array}\right)}+1328 \cdot q\left(\begin{array}{lll}
3 & 0 & \frac{1}{2} \\
0 & 1 & \frac{1}{2} \\
\frac{1}{2} & \frac{1}{2} & 1
\end{array}\right) \\
-1008 \cdot q^{\left(\begin{array}{lll}
2 & 0 & 0 \\
0 & 1 & 0 \\
0 & 0 & 1
\end{array}\right)}-131776 \cdot q^{\left(\begin{array}{lll}
2 & 1 & 1 \\
1 & 2 & 1 \\
1 & 1 & 2
\end{array}\right)}-6816512 \cdot q^{\left(\begin{array}{lll}
2 & 0 & 0 \\
0 & 2 & 0 \\
0 & 0 & 2
\end{array}\right)}+\cdots .
\end{gathered}
$$

The purpose of this note is to show that at each critical point $s$ one can provide the explicit rational number $R(s)$ and power of $\pi$ such that

$$
L\left(s, F_{12}, \mathrm{St}\right)=R(s) \pi^{\alpha_{s}}\langle\Delta, \Delta\rangle\left\langle g_{20}, g_{20}\right\rangle,
$$

where $\Delta$ is Ramanujan's discriminant cusp form and $g_{20}$ is the cusp form of weight 20 of level 1. 
The functional equation for a standard $L$ function in general case is proved by Böcherer [1]. For $F_{12}$ it is as follows:

$$
\Psi\left(s, F_{12}, \mathrm{St}\right)=\Psi\left(1-s, F_{12}, \mathrm{St}\right),
$$

where

$$
\begin{gathered}
\Psi\left(s, F_{12}, \mathrm{St}\right)=\gamma(s) L\left(s, F_{12}, \mathrm{St}\right) \\
\gamma(s)=C 2^{-3 s} \pi^{-7 s / 2} \Gamma\left(\frac{s+1}{2}\right) \Gamma(s+9) \Gamma(s+10) \Gamma(s+11)
\end{gathered}
$$

with some non-zero constant $C$. Critical points for $L\left(s, F_{12}, \mathrm{St}\right)$ in the sense of Deligne [4] are $s \in\{-8,-6,-4,-2,0,1,3,5,7,9\}$. In particular, gamma factor $\gamma(s)$ on the left hand side of the functional equation and $\gamma(1-s)$ on the right hand side is finite at these points.

Due to Miyawaki and Ikeda

$$
L\left(s, F_{12}, \mathrm{St}\right)=L(s+11, \Delta \otimes \Delta) L\left(s+10, g_{20}\right) L\left(s+9, g_{20}\right) .
$$

This theorem is the starting point of our investigation. We compute the result in two steps: first, compute $L(s+11, \Delta \otimes \Delta)$ for all points we are interested in, and then compute $L\left(s+10, g_{20}\right) L\left(s+9, g_{20}\right)$. We use RankinSelberg method to represent the $L$-funcion of multiplicative convolution as an integral over a fundamental domain, which can be written in terms of the Petersson scalar product. And, in order to compute it, we use a holomorphic projection.

To our knowledge this is the first example of a standard $L$-function of Siegel cusp form of degree 3, when the special values can be computed explicitly. The case of spinor $L$-function was treated in our other work [2]. The authors are very grateful to Alexei Panchishkin for intensive and encouraging discussions, this work was undertaken due to his keen interest into the subject.

\section{Generalities and notation}

Let $\mathfrak{H}=\{z \in \mathbb{C} \mid \Im(z)>0\}$ be the upper half-plane. For a positive integer $k$ and a Dirichlet character $\chi$ modulo a positive integer $N$ such that $\chi(-1)=(-1)^{k}$, we denote by $\mathcal{M}_{k}\left(\Gamma_{0}(N), \chi\right)$ the vector space of all holomorphic modular forms $f(z)$ of weight $k$ satisfying

$$
f(\gamma(z))=\chi(d)(c z+d)^{k} f(z) \text { for all } \gamma=\left(\begin{array}{ll}
a & b \\
c & d
\end{array}\right) \in \Gamma_{0}(N),
$$

where the variable $z \in \mathfrak{H}, \gamma(z)=\frac{a z+b}{c z+d}$, and

$$
\Gamma_{0}(N)=\left\{\left(\begin{array}{ll}
a & b \\
c & d
\end{array}\right) \in \mathrm{SL}_{2}(\mathbb{Z}) \mid c \equiv 0 \quad \bmod N\right\} \text {. }
$$


We denote by $S_{k}(N, \chi)$ the subspace of $\mathcal{M}_{k}\left(\Gamma_{0}(N), \chi\right)$ consisting of all cusp forms. Every element $f$ of $\mathcal{M}_{k}\left(\Gamma_{0}(N), \chi\right)$ has a Fourier expansion

$$
f(z)=\sum_{n=0}^{\infty} a(n) q^{n}
$$

where $q=\exp (2 \pi i z)$ and $a(n)$ are complex numbers in general.

The $L$-function associated to $f$ is defined as

$$
L(s, f)=\sum_{n=1}^{\infty} a(n) n^{-s} .
$$

More generally, with an arbitrary Dirichlet character $\omega$, the twisted $L$ function is defined as $L(s, f, \omega)=\sum_{n=1}^{\infty} a(n) \omega(n) n^{-s}$. These $L$-functions can be also written in the form of Euler product:

$$
\begin{aligned}
L(s, f) & =\prod_{p \text { prime }}\left(1-a(p) p^{-s}+\chi(p) p^{k-1-2 s}\right)^{-1}, \\
L(s, f, \omega) & =\prod_{p \text { prime }}\left(1-a(p) \omega(p) p^{-s}+\chi(p) \omega(p)^{2} p^{k-1-2 s}\right)^{-1} .
\end{aligned}
$$

Next, the Dirichlet $L$-series for any character $\chi$ of conductor $N$ is given by

$$
L(s, \chi)=\sum_{n=1}^{\infty} \chi(n) n^{-s}
$$

and its Euler product

$$
L(s, \chi)=\prod_{p \nmid N} \frac{1}{1-\chi(p) p^{-s}} .
$$

In the case, when $\chi$ is the identity Dirichlet character, the latter series is Riemann's zeta function $\zeta(s)=\sum_{n=1}^{\infty} \frac{1}{n^{s}}$.

Let $g(z)=\sum_{n=0}^{\infty} b(n) q^{n} \in \mathcal{M}_{l}\left(\Gamma_{0}(N), \xi\right)$ be another modular form of weight $l$ with Fourier coefficients $b(n)$. The $L$ function associated to two modular forms $f$ and $g$ is given by the additive convolution

$$
L(s, f, g)=\sum_{n=1}^{\infty} a(n) b(n) n^{-s} .
$$

Another type of $L$-functions associated to two modular forms is so-called Rankin's product $L$-function (multiplicative convolution). It is denoted by $L(s, f \otimes g)$ and defined as (see [14, page 786]):

$$
L(s, f \otimes g)=L_{N}(2 s+2-k-l, \chi \xi) L(s, f, g),
$$


where $L$-function $L_{N}(s, \omega)$ with a Dirichlet character $\omega$ modulo $N$ is defined, as usual, in $(2.1)$ with $\omega(n)=0$ for $(n, N) \neq 1$, and the Euler factors in (2.2), corresponding to the prime divisors of a number $N$, have been omitted. Note, that in the case, when $f$ and $g$ are cusp eigenforms, the lefthand side of (2.3) is an Euler product of degree 4 in view of the following Lemma:

Lemma 2.1 (Lemma 1, [14]). Suppose we have formally

$$
\sum_{n=1}^{\infty} A(n) n^{-s}=\prod_{p}\left[\left(1-\alpha_{p} p^{-s}\right)\left(1-\alpha_{p}^{\prime} p^{-s}\right)\right]^{-1}
$$

and

$$
\sum_{n=1}^{\infty} B(n) n^{-s}=\prod_{p}\left[\left(1-\beta_{p} p^{-s}\right)\left(1-\beta_{p}^{\prime} p^{-s}\right)\right]^{-1}
$$

Then

$$
\begin{aligned}
& \sum_{n=1}^{\infty} A(n) B(n) n^{-s}= \\
& \prod_{p} \frac{1-\alpha \alpha^{\prime} \beta \beta^{\prime} p^{-2 s}}{\left(1-\alpha \beta p^{-s}\right)\left(1-\alpha \beta^{\prime} p^{-s}\right)\left(1-\alpha^{\prime} \beta p^{-s}\right)\left(1-\alpha^{\prime} \beta^{\prime} p^{-s}\right)} .
\end{aligned}
$$

In the case when $f=g$, we introduce the convoluted zeta-function of $f$ with itself by defining Euler $p$-factor as

$$
\begin{aligned}
L_{p}(s, f \otimes f) & =\left(1-\alpha_{1}^{2} p^{-s}\right)^{-1}\left(1-\alpha_{1} \alpha_{2} p^{-s}\right)^{-1}\left(1-\alpha_{2}^{2} p^{-s}\right)^{-1} \\
& =\left(1-p^{-s+k-1}\right)^{-1}\left(1-\alpha_{1}^{2} p^{-s}\right)^{-1}\left(1-\alpha_{2}^{2} p^{-s}\right)^{-1} .
\end{aligned}
$$

Then the zeta-function $L(s, f \otimes f)$ is defined by

$$
L(s, f \otimes f)=\prod_{p} L_{p}(s, f \otimes f) .
$$

Therefore, it is related to the additive convolution by

$$
L(s, f \otimes f)=\frac{\zeta(2 s+2-2 k)}{\zeta(s+1-k)} \sum_{n=1}^{\infty} a^{2}(n) n^{-s} .
$$

For two elements $f, h \in \mathcal{M}_{k}\left(\Gamma_{0}(N)\right)$ such that the product $f h$ is a cusp form, the Petersson inner product $\langle f, h\rangle$ is defined as

$$
\langle f, h\rangle=\frac{1}{\left[\mathrm{SL}_{2}(\mathbb{Z}): \Gamma_{0}(N)\right]} \int_{\Phi_{N}} \overline{f(z)} h(z) y^{k-2} d x d y,
$$

where $z=x+i y, \Phi_{N}$ is a fundamental domain for $\mathfrak{H}$ modulo $\Gamma_{0}(N)$ and the bar denotes the complex conjugate. We also define $\langle f, h\rangle$ by $(2.5)$ for nearly holomorphic modular forms $f$ and $h$ on $\mathfrak{H}$ whenever the integral is 
convergent (see [15, section 8.2] for definition and properties of the nearly holomorphic modular forms).

We also briefly recall the definition of standard $L$-function for modular forms of arbitrary genus. Let $f \in \mathcal{M}_{k}^{n}(N, \psi)$ be an eigenfunction of all Hecke operators $f \longmapsto f \mid T, T \in \mathcal{L}_{q}^{n}(N)$ with $q$ being prime number, $q \nmid N$, so that $f \mid T=\lambda_{f}(T) f$. Then the number $\lambda_{f}(T) \in \mathbb{C}$ define a homomorphism $\lambda_{f}: \mathcal{L} \longrightarrow \mathbb{C}$ which is uniquely determined by $(n+1)$-tuple of numbers

$$
\left(\alpha_{0}, \alpha_{1}, \ldots, \alpha_{n}\right)=\left(\alpha_{0, f}(q), \alpha_{1, f}(q), \ldots, \alpha_{n, f}(q)\right) \in\left[(\mathbb{C})^{n+1}\right]^{W_{n}}
$$

which are called the Satake $q$-parameters of the modular form $f$.

Now let the variables $T_{0}, T_{1}, \ldots, T_{n}$ be equal to the corresponding Satake $q$-parameters $\alpha_{0, f}(q), \alpha_{1, f}(q), \ldots, \alpha_{n, f}(q)$ then

$$
R_{f, q}(z)=\prod_{i=1}^{n}\left(1-\alpha_{i}^{-1} z\right)\left(1-\alpha_{i} z\right) \in \mathbb{Q}\left[\alpha_{0}^{ \pm 1}, \ldots, \alpha_{n}^{ \pm 1}\right] .
$$

The standard zeta function of $f$ is defined by means of the Satake $p$ parameters as the following Euler product:

$$
L(s, f, \mathrm{St}, \chi)=\prod_{q \nmid N} L^{(q)}(s, f, \mathrm{St}, \chi)
$$

with

$$
\begin{aligned}
& L^{(q)}(s, f, \text { St }, \chi)=\left(1-\chi(q) \psi(q) q^{-s}\right)^{-1} R_{f, q}\left(\chi(q) \psi(q) q^{-s}\right)^{-1}, \\
& L(s, f, \text { St }, \chi)= \\
& \quad \prod_{p}\left(\left(1-\frac{\chi(p)}{p^{s}}\right) \prod_{i=1}^{m}\left(1-\frac{\chi(p) \alpha_{i}(p)}{p^{s}}\right)\left(1-\frac{\chi(p) \alpha_{i}(p)^{-1}}{p^{s}}\right)\right)^{-1} .
\end{aligned}
$$

\section{Computation of $L(s, \Delta \otimes \Delta)$}

The first term $L(s+11, \Delta \otimes \Delta)$ is the symmetric square of cusp form $\Delta$ by the mean of (2.3). This $L$ function has been already studied in details by Rankin, Zagier, Li, Sturm and others. Using formulas from [12] and [17, page 116] we have the values of $L(s, \Delta \otimes \Delta)$ at the points $s \in$ $\{12,14,16,18,20\}$. The values at points $s \in\{3,5,7,9,11\}$ can be obtained from the functional equation (see [8])

$$
D^{*}(s, \Delta)=D^{*}(23-s, \Delta),
$$

where

$$
D^{*}(s, \Delta)=2^{-s} \pi^{-3 s / 2} \Gamma(s) \Gamma\left(\frac{s-10}{2}\right) L(s, \Delta \otimes \Delta) .
$$

The results available online in supplementary materials [5, Section 1]. 


\section{The expression for $L\left(s, g_{20}\right) L\left(s-1, g_{20}\right)$}

We compute the product $L\left(s, g_{20}\right) L\left(s-1, g_{20}\right)$ at points critical points of $L\left(s, F_{12}\right.$, St) (note the shift by 10$) s \in\{2,4,6,8,10,11,13,15,17,19\}$ in two steps. The idea is to present the product $L(s, f) L(s-1, f)$ as an $L$ function of the Rankin convolution of $f$ with appropriate Eisenstein series and to use Rankin-Selberg method to relate the obtained expression to the Petersson inner product.

Let $g_{20} \in S_{20}$ be the cusp form of weight 20 of level 1 :

$$
\begin{aligned}
g_{20}(z) & =\sum_{n=1}^{\infty} a(n) q^{n} \\
& =q+456 q^{2}+50652 q^{3}-316352 q^{4}+2377410 q^{5}-\cdots
\end{aligned}
$$

and its associated $L$-function is

$$
L\left(s, g_{20}\right)=\sum_{n=1}^{\infty} a(n) n^{-s}
$$

Assume $1-a(p) X+p^{19} X^{2}=\left(1-\alpha_{p} X\right)\left(1-\alpha_{p}^{\prime} X\right)$, then $\alpha_{p}+\alpha_{p}^{\prime}=a(p)$, $\alpha_{p} \alpha_{p}^{\prime}=p^{19}$, and

$$
L\left(s, g_{20}\right)=\prod_{p}\left(\left(1-\alpha_{p} p^{-s}\right)\left(1-\alpha_{p}^{\prime} p^{-s}\right)\right)^{-1}
$$

Let

$$
\begin{aligned}
G_{2}(z) & =-\frac{1}{24}+\sum_{n=1}^{\infty} \sigma_{1}(n) q^{n} \\
& =-\frac{1}{24}+q+3 q^{2}+4 q^{3}+7 q^{4}+\cdots
\end{aligned}
$$

where $\sigma_{1}(n)=\sum_{d \mid n} d$ is the divisor function defined as the sum of the divisors of $n$.

Consider the Eisenstein series (see [9, Corollary 7.2.14])

$$
G_{2, p}(z)=G_{2}(z)-p G_{2}(p z)=\frac{p-1}{24}+\sum_{n=1}^{\infty} \sum_{d \mid n, p \nmid d} d q^{n},
$$


of weight 2 for $\Gamma_{0}(p)$ and the corresponding $L$ series

$$
\begin{aligned}
L\left(s, G_{2, p}\right) & =\sum_{\substack { n=1 \\
\begin{subarray}{c}{p \nmid d \\
p \nmid d{ n = 1 \\
\begin{subarray} { c } { p \nmid d \\
p \nmid d } }\end{subarray}}^{\infty} d n^{-s} \\
& =\sum_{\substack{d, d_{1} \geqslant 1 \\
p \nmid d}} d\left(d d_{1}\right)^{-s} \\
& =\sum_{\substack{d \geqslant 1 \\
p \nmid d}} d^{1-s} \sum_{d_{1} \geqslant 1} d_{1}^{-s} \\
& =\left(1-p^{1-s}\right) \zeta(s-1) \zeta(s) .
\end{aligned}
$$

Let us put $p=2$, then consider the following holomorphic modular form of weight 2 and level 2

$$
\begin{aligned}
G_{2,2}(z) & =G_{2}(z)-2 G_{2}(2 z) \\
& =\frac{1}{24}+q+q^{2}+4 q^{3}+q^{4}+6 q^{5}+4 q^{6}+\cdots
\end{aligned}
$$

and

$$
L\left(s, G_{2,2}\right)=\left(1-2^{1-s}\right) \zeta(s-1) \zeta(s)
$$

Note,

$$
G_{2,2}=\sum_{n=0}^{\infty} b(n) q^{n} \in \mathcal{M}_{2}\left(\Gamma_{0}(2), \xi\right)
$$

with

$$
\xi(n)=(1 \bmod 2)(n)= \begin{cases}1, & \text { if } n \text { odd } \\ 0, & \text { if } n \text { even }\end{cases}
$$

Similarly to (4.1), consider Fourier coefficients and the decomposition

$$
\begin{aligned}
L\left(s, G_{2,2}\right) & =\sum_{n=1}^{\infty} b(n) n^{-s} \\
& =\left(1-2^{1-s}\right) \zeta(s-1) \zeta(s) \\
& =\left(1-2^{1-s}\right) \prod_{p}\left(\left(1-p^{1-s}\right)\left(1-p^{-s}\right)\right)^{-1} \\
& =\prod_{p}\left(\left(1-\beta_{p} p^{-s}\right)\left(1-\beta_{p}^{\prime} p^{-s}\right)\right)^{-1},
\end{aligned}
$$

where $\beta(p)=1$ for all $p, \beta^{\prime}(2)=0$ and $\beta^{\prime}(p)=p$ for all odd primes. 
Recall Lemma 2.1 and consider $g_{20}$ and $G_{2,2}$ in its context. By definition (2.3) and using Lemma 2.1 with $k=20, l=2, N=2, \chi=1$ we obtain

$$
\begin{aligned}
& L\left(s, g_{20} \otimes G_{2,2}\right)=L_{2}(2 s+2-20-2, \psi) L\left(s, g_{20}, G_{2,2}\right) \\
& =\prod_{p \neq 2}\left(1-p^{20-2 s}\right)^{-1} \cdot \sum_{n=1}^{\infty} a(n) b(n) n^{-s} \\
& =\prod_{p \neq 2}\left(1-p^{20-2 s}\right)^{-1} \\
& \times \prod_{p} \frac{1-\alpha_{p} \alpha_{p}^{\prime} \beta_{p} \beta_{p}^{\prime} p^{-2 s}}{\left(1-\alpha_{p} \beta_{p} p^{-s}\right)\left(1-\alpha_{p}^{\prime} \beta_{p} p^{-s}\right)\left(1-\alpha_{p} \beta_{p}^{\prime} p^{-s}\right)\left(1-\alpha_{p}^{\prime} \beta_{p}^{\prime} p^{-s}\right)} \\
& =\frac{1}{\left(1-\alpha_{2} 2^{-s}\right)\left(1-\alpha_{2}^{\prime} 2^{-s}\right)} \\
& \times \prod_{p \neq 2} \frac{1}{\left(1-\alpha_{p} p^{-s}\right)\left(1-\alpha_{p}^{\prime} p^{-s}\right)\left(1-\alpha_{p} p^{1-s}\right)\left(1-\alpha_{p}^{\prime} p^{1-s}\right)} \\
& =\prod_{p} \frac{1}{\left(1-\alpha_{p} p^{-s}\right)\left(1-\alpha_{p}^{\prime} p^{-s}\right)} \prod_{p \neq 2} \frac{1}{\left(1-\alpha_{p} p^{1-s}\right)\left(1-\alpha_{p}^{\prime} p^{1-s}\right)} \\
& =L\left(s, g_{20}\right) L\left(s-1, g_{20}\right)\left(1-a(2) 2^{1-s}+2^{19} 2^{2-2 s}\right) \\
& =\left(1-456 \cdot 2^{1-s}+2^{21-2 s}\right) L\left(s, g_{20}\right) L\left(s-1, g_{20}\right) \text {. }
\end{aligned}
$$

Finally, we obtain the following identity

$$
L\left(s, g_{20}\right) L\left(s-1, g_{20}\right)=\frac{L\left(s, g_{20} \otimes G_{2,2}\right)}{\left(1-456 \cdot 2^{1-s}+2^{21-2 s}\right)} .
$$

\section{Computation of $L\left(s, g_{20} \otimes G_{2,2}\right)$}

Now we express $L\left(s, g_{20} \otimes G_{2,2}\right)$ (at integer points) as a multiple of Petersson inner product $\left\langle g_{20}, g_{20}\right\rangle$. Put $g_{20}^{*}(z)=\overline{g_{20}(-\bar{z})}=\sum \overline{a(n)} q^{n}$. We use Shimura's formula $[14,(2.4)]$

$$
\begin{aligned}
L\left(s, g_{20} \otimes G_{2,2}\right) & \\
= & \frac{(4 \pi)^{s}}{2 \Gamma(s)} \int_{\Phi_{2}} \overline{g_{20}^{*}(z)} G_{(2,2)}(z) E_{18,2}(z, s-19, \xi) y^{s-1} d x d y \\
& =\frac{(4 \pi)^{s}}{2 \Gamma(s)} \int_{\Phi_{2}} \overline{g_{20}^{*}(z)} G_{(2,2)}(z) E_{18,2}(z, s-19, \xi) y^{s-19} y^{18} d x d y \\
& =\frac{(4 \pi)^{s}\left[\mathrm{SL}_{2}(\mathbb{Z}): \Gamma_{0}(2)\right]}{2 \Gamma(s)}\left\langle g_{20}(z), G_{2,2}(z) y^{19} E_{18,2}(z, s-19, \xi)\right\rangle \\
& =\frac{3}{2} \frac{(4 \pi)^{s}}{\Gamma(s)}\left\langle g_{20}(z), \mathcal{H o l}\left(G_{2,2}(z) y^{19} E_{18,2}(z, s-19, \xi)\right)\right\rangle
\end{aligned}
$$


where $\langle f, g\rangle$ is the Petersson inner product (2.5), $\Phi_{2}$ denotes a fundamental domain for $\Gamma_{0}(2) \backslash \mathfrak{H}, z=x+i y$,

$$
E_{\lambda, N}(z, s, \xi)=\sum_{(m, n)}^{\prime} \xi(n)(m N z+n)^{-\lambda}|m N z+n|^{-2 s},
$$

$\sum^{\prime}$ denotes the summation over all $(m, n) \in \mathbb{Z}^{2},(m, n) \neq(0,0), \mathcal{H o l}(F)$ is the operator of holomorphic projection (see $[3,(2.148)]$ ). It is defined so, that $\langle f, F\rangle=\langle f, \mathcal{H o l}(F)\rangle$ for all $f \in S_{k}(N, \psi)$.

In order to compute the holomorphic projection of the product in the last identity of (5.1) $\mathcal{H o l}\left(G_{2,2}(z)(4 \pi y)^{s+1-k} E_{k-2,2}(z, s+1-k, \xi)\right)$ we need the explicit Fourier coefficients of the product $G_{2,2} E_{k-2,2}$. The holomorphic projection belongs to the space of cups forms of weight 20 and level 2 . It is a 4-dimensional space, therefore we need at least four Fourier coefficients of it in order to identify it in a fixed basis. The Fourier coefficient of $G_{2,2}(z)$ are known. The Fourier expansion for $E_{k-2,2}(z, s+1-k, \xi)$ can be obtained in a convenient form using the Whittaker functions by applying the following proposition:

Proposition 5.1 (Proposition 2.2, [11]). Suppose that $s, l \in \mathbb{Z}$ are two integers satisfying $s \leqslant 0$ and $s+l>0$, then there is the following Fourier expansion:

$$
\begin{aligned}
& \mathbf{E}_{l, N}(z, s ; a, b)=\delta\left(\frac{a}{N}\right)\left[\zeta(l+2 s ; b, N)+(-1)^{l+2 s} \zeta(l+2 s ;-b, N)\right] \\
& +\frac{(-2 \pi i)^{l+2 s}(-1)^{s} \Gamma(l+2 s-1)}{(4 \pi y)^{l+2 s-1} N \Gamma(l+s) \Gamma(s)} \\
& \quad \times\left[\zeta(l+2 s-1 ; a, N)+(-1)^{l+2 s} \zeta(l+2 s-1 ;-a, N)\right] \\
& +\frac{(-2 \pi i)^{l+2 s}(-1)^{s}}{N^{l+2 s} \Gamma(l+s)} \\
& \quad \times\left(\sum_{\substack{d d^{\prime}>0 \\
d^{\prime} \equiv a \bmod N}} \operatorname{sgn}(d) d^{l+2 s-1} e^{\frac{2 \pi i d b}{N}} W\left(\frac{4 \pi d d^{\prime} y}{N}, l+s, s\right) e^{\frac{2 \pi i d d^{\prime} z}{N}}\right),
\end{aligned}
$$

where $\delta(x)=1$ if $x$ is an integer, $\delta(x)=0$ otherwise, and

$$
\zeta(s ; a, N)=\sum_{0<n \equiv a(\bmod N)} n^{-s}
$$

denote the partial Riemann zeta function (defined if necessary by analytic continuation on $s$ ).

In this Proposition the Eisenstein series $\mathbf{E}_{l, N}$ of weight $l$ are defined as

$$
\mathbf{E}_{l, N}(z, s ; a, b)=\sum(c z+d)^{-l}|c d+d|^{-2 s},
$$


where $(c, d) \equiv(a, b)(\bmod N)$ and $(c, d) \neq(0,0), a, b \in \mathbb{Z} / N \mathbb{Z}$.

In order to apply the above proposition for our case, we note that

$$
\begin{gathered}
E_{18,2}(z, s+19, \xi)=\mathbf{E}_{18,2}(z, s-19,0,1), \\
k=20, \quad 1<s \leqslant 19, \quad a=0, \quad b=1, \quad N=2, \quad l=18, \\
\delta\left(\frac{a}{N}\right)=1, \\
\zeta(s ; 0,2)=\sum_{0<n \equiv 0(2)} n^{-s}=\sum_{n=1}^{\infty}(2 n)^{-s}=2^{-s} \zeta(s), \\
\zeta(s ; 1,2)=\sum_{0<n \equiv 1(2)} n^{-s}=\zeta(s)-\zeta(s ; 0,2)=\left(1-2^{-s}\right) \zeta(s) .
\end{gathered}
$$

The Whittaker function $W(y, \alpha, \beta)$ is defined as

$$
W(y, \alpha, \beta)=\Gamma(\beta)^{-1} \int_{0}^{+\infty}(u+1)^{\alpha-1} u^{\beta-1} \mathrm{e}^{-y u} d u
$$

for $y>0, \alpha, \beta \in \mathbb{C}$ with $\Re(\beta)>0$ and for arbitrary $\alpha$ and $\beta$ this function is defined by the analytic continuation and the functional equation:

$$
W(y, \alpha, \beta)=y^{1-\alpha-\beta} W(y, 1-\beta, 1-\alpha) .
$$

For a non negative integer $r$, we have

$$
W(y, \alpha,-r)=\sum_{i=0}^{r} \frac{(-1)^{i}\left(\begin{array}{l}
r \\
i
\end{array}\right) \Gamma(\alpha)}{\Gamma(\alpha-i)} y^{r-i} .
$$

Now we write the explicit expansion for the even weight $l=k-2$ (when $k$ is even) at the point $s+1-k$ :

$$
\begin{aligned}
(4 \pi y)^{s+1-k} & E_{k-2,2}(z, s+1-k, \xi) \\
=(4 \pi y)^{s+1-k}(2 \zeta(k-2+2(s+1-k) ; 1,2) & \\
+ & \frac{(-2 \pi i)^{k-2+2(s+1-k)}(-1)^{s+1-k} \Gamma(k-2+2(s+1-k)-1)}{(4 \pi y)^{k-2+2(s+1-k)-1} 2 \Gamma(k-2+s+1-k) \Gamma(s+1-k)} \\
& \times 2 \zeta(k-2+2(s+1-k)-1 ; 0,2) \\
+ & \frac{(-2 \pi i)^{k-2+2(s+1-k)}(-1)^{s+1-k}}{2^{k-2+2(s+1-k)} \Gamma(k-2+s+1-k)} \\
& \times \sum_{d^{\prime} \equiv 0 \bmod 2} \operatorname{sgn}(d) d^{k-2+2(s+1-k)-1} e^{\pi i d} \\
& \left.\times W\left(\frac{4 \pi d d^{\prime} y}{2}, k-2+s+1-k, s+1-k\right) e^{\pi i d d^{\prime} z}\right) .
\end{aligned}
$$


The first term of (5.2) becomes

$$
\begin{aligned}
(4 \pi y)^{s+1-k} 2 \zeta(k-2+2(s+1- & k) ; 1,2) \\
& =2(4 \pi y)^{s+1-k}\left(1-2^{k-2 s}\right) \zeta(2 s-k) .
\end{aligned}
$$

The second term of (5.2) becomes

$$
\begin{aligned}
(4 \pi y)^{s+1-k} \frac{(2 \pi i)^{2 s-k}(-1)^{s+1-k} \Gamma(2 s-k-1)}{(4 \pi y)^{2 s-k-1} 2 \Gamma(s-1) \Gamma(s+1-k)} 2 \zeta(2 s-k-1 ; 0,2) \\
=(4 \pi y)^{2-s} \frac{(2 \pi)^{2 s-k}(-1)^{s-k / 2}(-1)^{s+1-k} \Gamma(2 s-k-1)}{\Gamma(s-1) \Gamma(s+1-k)} \\
\quad \times 2^{k+1-2 s} \zeta(2 s-k-1) \\
=(4 \pi y)^{2-s} \frac{2 \pi^{2 s-k}(-1)^{1-k / 2} \Gamma(2 s-k-1) \zeta(2 s-k-1)}{\Gamma(s-1) \Gamma(s+1-k)}
\end{aligned}
$$

The last term of (5.2) contains the sum over all $d$ and $d^{\prime}$ such that the product $d d^{\prime}$ is positive and $d^{\prime} \equiv 0(\bmod 2)$. Let us write $d^{\prime}=2 d_{1}$ and break down the summation in two parts, one for positive $d$ and another for negative $d$. We also substitute $d d_{1}$ by $n$. Therefore,

$$
\begin{aligned}
& \sum_{\substack{d d^{\prime}>0 \\
d^{\prime}}} \operatorname{sgn}(d) d^{2 s-k-1} e^{\pi i d} W\left(2 \pi d d^{\prime} y, s-1, s+1-k\right) e^{\pi i d d^{\prime} z} \\
= & \sum_{\substack{d>0 \\
d_{1}>0}} d^{2 s-k-1}(-1)^{d} W\left(4 \pi d d_{1} y, s-1, s+1-k\right) e^{2 \pi i d d_{1} z} \\
& +\sum_{\substack{d<0 \\
d_{1}<0}}(-1) d^{2 s-k-1}(-1)^{d} W\left(4 \pi d d_{1} y, s-1, s+1-k\right) e^{2 \pi i d d_{1} z} \\
= & \sum_{n>0} \sum_{d \mid n} d^{2 s-k-1}(-1)^{d} W(4 \pi n y, s-1, s+1-k) q^{n} \\
& +\sum_{\substack{d>0 \\
d_{1}>0}}(-1)(-1)^{2 s-k-1} d^{2 s-k-1}(-1)^{d} W\left(4 \pi d d_{1} y, s-1, s+1-k\right) e^{2 \pi i d d_{1} z} \\
= & \sum_{n>0} \sum_{d \mid n} d^{2 s-k-1}(-1)^{d} W(4 \pi n y, s-1, s+1-k) q^{n} \\
& +\sum_{n>0} \sum_{d \mid n} d^{2 s-k-1}(-1)^{d} W(4 \pi n y, s-1, s+1-k) q^{n} \\
= & 2 \sum_{n>0} \sum_{d \mid n} d^{2 s-k-1}(-1)^{d} W(4 \pi n y, s-1, s+1-k) q^{n} .
\end{aligned}
$$


The last term of (5.2) becomes

$$
\frac{(-1)^{1-k / 2} 2 \pi^{2 s-k}}{\Gamma(s-1)} \sum_{n>0} \sum_{d \mid n}(-1)^{d} d^{2 s-k-1} W(4 \pi n y, s-1, s+1-k) q^{n} .
$$

To abbreviate the following manipulations we introduce some notations. Let

$$
\begin{aligned}
& C_{0}^{\prime}(s)=\frac{(-1)^{1-k / 2} 2 \pi^{2 s-k} \Gamma(2 s-k-1) \zeta(2 s-k-1)}{\Gamma(s-1) \Gamma(s+1-k)} \\
& C_{0}^{\prime \prime}(s)=2\left(1-2^{k-2 s}\right) \zeta(2 s-k) \\
& C_{1}(s)=(-1)^{k / 2} 2 \pi^{2 s-k} \\
& C_{2}(s)=(-1)^{k / 2}\left(2-2^{2 s-k}\right) \pi^{2 s-k} \\
& C_{3}(s)=(-1)^{k / 2} 2\left(1+3^{2 s-k-1}\right) \pi^{2 s-k} \\
& C_{4}(s)=(-1)^{k / 2}\left(2-2^{2 s-k}-2^{4 s-2 k-1}\right) \pi^{2 s-k}
\end{aligned}
$$

then (5.2) can be written as

$$
\begin{aligned}
(4 \pi y)^{s+1-k} E_{k-2,2}(z, s+1-k, \xi) & \\
= & C_{0}^{\prime}(4 \pi y)^{2-s}+C_{0}^{\prime \prime}(4 \pi y)^{s+1-k} \\
& +C_{1} \frac{W(4 \pi y, s-1, s+1-k)}{\Gamma(s-1)}(4 \pi y)^{s+1-k} q \\
& +C_{2} \frac{W(8 \pi y, s-1, s+1-k)}{\Gamma(s-1)}(4 \pi y)^{s+1-k} q^{2} \\
& +C_{3} \frac{W(12 \pi y, s-1, s+1-k)}{\Gamma(s-1)}(4 \pi y)^{s+1-k} q^{3} \\
& +C_{4} \frac{W(16 \pi y, s-1, s+1-k)}{\Gamma(s-1)}(4 \pi y)^{s+1-k} q^{4}+\cdots
\end{aligned}
$$

To find the image of the projection operator as a $q$-expansion $\mathcal{H o l}(F)=$ $\sum A_{n}(s) q^{n}$ we apply the Holomorphic Projection Lemma [6, Proposition (5.1)] (the Lemma is originally due to Sturm [16]). We denote the Fourier coefficients of the product inside of the holomorphic projection operator by $\widetilde{A}_{n}(s, y)$ :

$$
F(z, s, y)=G_{2,2}(z)(4 \pi y)^{s+1-k} E_{k-2,2}(z, s+1-k, \xi)=\sum \widetilde{A}_{n}(s, y) q^{n} .
$$

It should be noted, that the relevant polynomial decay hypotheses of the Lemma is satisfied for all actions on $E_{k-2,2}(z, s+1-k, \xi) \mid \gamma$ of $\gamma \in \mathrm{SL}_{2}(\mathbb{Z})$ and at each critical point $s$, see $[11,(2.3)]$.

Consider the case of weight $k=20$. Recall that the holomorphic projection belongs to the space of cups forms of weight 20 and level 2, which is a 4-dimensional space. This space can be spanned by the the following four 
modular forms: $g_{20}(z), g_{20}(2 z)$ and the orthogonal subspace of newforms $h_{20,2}^{(1)}(z)$ and $h_{20,2}^{(2)}(z)$ :

$$
\begin{aligned}
g_{20}(z) & =q+456 q^{2}+50652 q^{3}-316352 q^{4}-2377410 q^{5}+\cdots, \\
g_{20}(2 z) & =q^{2}+456 q^{4}+50652 q^{6}-316352 q^{8}+\cdots, \\
h_{20,2}^{(1)}(z) & =q-512 q^{2}-13092 q^{3}+262144 q^{4}+6546750 q^{5}+\cdots \\
h_{20,2}^{(2)}(z) & =q+512 q^{2}-53028 q^{3}+262144 q^{4}-5556930 q^{5}+\cdots
\end{aligned}
$$

Consider the linear combination

$$
\mathcal{H o l}(F)(z)=K_{1} g_{20}(z)+K_{2} g_{20}(2 z)+K_{3} h_{20,2}^{(1)}(z)+K_{4} h_{20,2}^{(2)}(z) .
$$

Coefficients $K_{i}=K_{i}(s)$ can be found explicitly by comparing the Fourier coefficients (we also do not indicate the dependence of $A_{i}$ from $s$ to abbreviate the notation):

$$
\left\{\begin{array}{l}
A_{1}=K_{1} \cdot 1+K_{2} \cdot 0+K_{3} \cdot 1+K_{4} \cdot 1 \\
A_{2}=K_{1} \cdot 456+K_{2} \cdot 1+K_{3} \cdot(-512)+K_{4} \cdot 512 \\
A_{3}=K_{1} \cdot 50652+K_{2} \cdot 0+K_{3} \cdot(-13092)+K_{4} \cdot(-53028) \\
A_{4}=K_{1} \cdot(-316352)+K_{2} \cdot 456+K_{3} \cdot 262144+K_{4} \cdot 262144
\end{array}\right.
$$

Resolving the system of linear equations (5.4), we obtain

$$
\left\{\begin{array}{l}
K_{1}=\frac{-13 A_{4}+152 A_{3}+5928 A_{2}+8432992 A_{1}}{22947840} \\
K_{2}=\frac{4229 A_{4}+24104 A_{3}+940056 A_{2}-311728736 A_{1}}{2868480} \\
K_{3}=\frac{3 A_{4}+16 A_{3}-1368 A_{2}+762432 A_{1}}{2039808} \\
K_{4}=\frac{-A_{4}-16 A_{3}+456 A_{2}+286144 A_{1}}{1105920}
\end{array}\right.
$$

Recall that the computation of the Fourier coefficients of the Holomorphic projection is given by the formula [6, Proposition (5.1)]:

$$
\begin{aligned}
A_{m}(s) & =\frac{(4 \pi m)^{k-1}}{(k-2) !} \int_{0}^{\infty} \widetilde{A}_{m}(s, y) \mathrm{e}^{-4 \pi m y} y^{k-2} d y \\
& =\frac{1}{(k-2) !} \int_{0}^{\infty} \widetilde{A}_{m}(s, y) \mathrm{e}^{-4 \pi m y}(4 \pi m y)^{k-2} d(4 \pi m y)
\end{aligned}
$$

The explicit computations of $A_{m}$ and $K_{m}$ are given in online supplementary materials [5, Section 2]. 


\section{Result for $L\left(s+10, g_{20}\right) L\left(s+9, g_{20}\right)$}

Recall that in each critical point $s \in\{-8,-6,-4,-2,0,1,3,5,7,9\}$ of the standard $L$-function $L\left(s, F_{12}, \mathrm{St}\right)$ according to (4.2) we compute two factors, one of which is $L\left(s+10, g_{20}\right) L\left(s+9, g_{20}\right)$. We consider the product $L\left(s, g_{20}\right) L\left(s-1, g_{20}\right)$ at points $s \in\{2,4,6,8,10,11,13,15,17,19\}$. In the Section 4 we established the formula

$$
L\left(s, g_{20}\right) L\left(s-1, g_{20}\right)=\frac{L\left(s, g_{20} \otimes G_{2,2}\right)}{1-456 \cdot 2^{1-s}+2^{21-2 s}} .
$$

Later, we found that

$$
L\left(s, g_{20} \otimes G_{2,2}\right)=\frac{3}{2} \frac{(4 \pi)^{19}}{\Gamma(s)}\left\langle g_{20}(z), \mathcal{H o l}(F(z, s, y))\right\rangle,
$$

where

$$
F(z, s, y)=G_{2,2}(z)(4 \pi y)^{s-19} E_{18,2}(z, s-19, \xi),
$$

We computed coefficients $K_{1}(s), K_{2}(s), K_{3}(s)$ and $K_{4}(s)$ so that

$$
\mathcal{H o l}(F)(z)=K_{1} g_{20}(z)+K_{2} g_{20}(2 z)+K_{3} h_{20,2}^{(1)}(z)+K_{4} h_{20,2}^{(2)}(z) .
$$

Both forms $h_{20,2}^{(1)}(z)$ and $h_{20,2}^{(2)}(z)$ are orthogonal to $g_{20}$, therefore their Petersson inner product with $g_{20}$ is zero. We compute the Petersson inner product $\left\langle g_{20}(z), g_{20}(2 z)\right\rangle$ using the trace operator (see [13, paragraph 3.1]).

Recall that if we identify a modular form $f$ of weight $k$ with its $q$ expansion, we have

$$
f(m z)=\left.f(z)\right|_{k} V(m)=\left.m^{-k / 2} f(z)\right|_{k}\left(\begin{array}{cc}
m & 0 \\
0 & 1
\end{array}\right)
$$

In our case $f(z)=g_{20}(z), k=20, m=2$. Consider $\gamma \in \Gamma_{0}(2) \backslash \mathrm{SL}_{2}(\mathbb{Z})$. The summation over all $\gamma$ gives

$$
\begin{aligned}
\left\langle g_{20}(z), g_{20}(2 z)\right\rangle & =2^{-10}\left[\Gamma: \Gamma_{0}(2)\right]^{-1} \sum_{\gamma}\left\langle g_{20}(z)\left|\gamma, g_{20}(z)\right|\left(\begin{array}{cc}
2 & 0 \\
0 & 1
\end{array}\right) \gamma\right\rangle \\
& =2^{-10} \cdot 3^{-1}\left\langle g_{20}(z), \operatorname{Tr}^{(2)}\left(g_{20}(z) \mid\left(\begin{array}{ll}
2 & 0 \\
0 & 1
\end{array}\right)\right)\right\rangle
\end{aligned}
$$

The trace operator $\operatorname{Tr}^{(\mathrm{N})}: \mathcal{M}_{k}\left(\Gamma_{0}(N)\right) \rightarrow \mathcal{M}_{k}\left(\mathrm{SL}_{2}(\mathbb{Z})\right)$ is defined by the action $\left.f \rightarrow \sum_{\gamma \in \Gamma_{0}(N) \backslash \mathrm{SL}_{2}(\mathbb{Z})} f\right|_{k} \gamma$. We have

$$
\operatorname{Tr}^{(2)}\left(g_{20}(z) \mid\left(\begin{array}{ll}
2 & 0 \\
0 & 1
\end{array}\right)\right)=2^{-9} T_{2}\left(g_{20}\right)
$$

where $T_{2}$ is the Hecke operator, therefore,

$$
\begin{aligned}
\left\langle g_{20}(z), g_{20}(2 z)\right\rangle & =2^{-10} \cdot 3^{-1} \cdot 2^{-9}\left\langle g_{20}(z), T_{2}\left(g_{20}(z)\right)\right\rangle \\
& =2^{-16} \cdot 19\left\langle g_{20}(z), g_{20}(z)\right\rangle .
\end{aligned}
$$

Therefore,

$$
\left\langle g_{20}(z), \mathcal{H o l}(F(z, s, y))\right\rangle=\left(K_{1}(s)+2^{-16} \cdot 19 \cdot K_{2}(s)\right)\left\langle g_{20}(z), g_{20}(z)\right\rangle
$$


The explicit values of the product $L\left(s+10, g_{20}\right) L\left(s+9, g_{20}\right)$ for each critical point $s$ are given in online supplementary materials [5, Section 3].

\section{The main identity}

Combining the above results into the original expression (1) we get the final result

$$
\begin{aligned}
& L\left(s, F_{12}, \mathrm{St}\right) \\
& \quad=L(s+11, \Delta \otimes \Delta) L\left(s+10, g_{20}\right) L\left(s+9, g_{20}\right) \\
& \quad=R_{s}^{(\Delta)} \pi_{s}^{(\Delta)} R_{s}^{\left(g_{20}\right)} \pi_{s}^{\left(g_{20}\right)}\langle\Delta, \Delta\rangle\left\langle g_{20}, g_{20}\right\rangle \\
& \quad=R_{s}^{(\Delta)} \pi_{s}^{(\Delta)} \frac{3}{2} \frac{(4 \pi)^{19}}{\Gamma(s+10)} \frac{\left(K_{1}(s)+2^{-16} \cdot 19 \cdot K_{2}(s)\right)}{1-456 \cdot 2^{-9-s}+2^{1-2 s}}\langle\Delta, \Delta\rangle\left\langle g_{20}, g_{20}\right\rangle .
\end{aligned}
$$

where $R_{s}^{(\Delta)}, \pi_{s}^{(\Delta)}$ were computed in Section 3 and $K_{1}(s), K_{2}(s)$ were computed in Section 6 .

For each critical values $s \in\{-8,-6,-4,-2,0,1,3,5,7,9\}$ we evaluate the final expression in the form

$$
L\left(s, F_{12}, \mathrm{St}\right)=R_{s} \pi_{s}\langle\Delta, \Delta\rangle\left\langle g_{20}, g_{20}\right\rangle
$$

with the rational coefficient $R_{s}$ factorised in primes and the corresponding power of $\pi$. We also provide approximate numerical values (Petersson inner products evaluation is provided in online supplementary materials [5, Section 4]). 


\begin{tabular}{|c|c|c|c|}
\hline$s$ & $R_{s}$ & $\pi_{s}$ & numerical value \\
\hline-8 & $\frac{-1 \cdot 2^{31} \cdot 17 \cdot 11411 \cdot 1207259}{3 \cdot 5^{2} \cdot 7 \cdot 11 \cdot 13 \cdot 61}$ & $\pi^{6}$ & -903525.807173 \\
\hline-6 & $\frac{-1 \cdot 2^{26} \cdot 47 \cdot 791797}{3^{6} \cdot 17 \cdot 113}$ & $\pi^{12}$ & -14105.832863 \\
\hline-4 & $\frac{2^{24} \cdot 392033}{3^{5} \cdot 5^{3} \cdot 7 \cdot 17 \cdot 19}$ & $\pi^{18}$ & 728.260808 \\
\hline-2 & $\frac{-1 \cdot 2^{26} \cdot 479903}{3^{8} \cdot 5^{3} \cdot 7^{3} \cdot 13 \cdot 17 \cdot 157}$ & $\pi^{24}$ & -24.122802 \\
\hline 0 & $\frac{2^{22} \cdot 5779}{3^{13} \cdot 5^{4} \cdot 7^{3} \cdot 11 \cdot 13}$ & $\pi^{30}$ & 3.485667 \\
\hline 1 & $\frac{2^{25} \cdot 2269}{3^{14} \cdot 5^{4} \cdot 7^{3} \cdot 11^{2} \cdot 13 \cdot 17}$ & $\pi^{34}$ & 1.901053 \\
\hline 3 & $\frac{2^{40}}{3^{16} \cdot 5^{6} \cdot 7^{4} \cdot 11^{3} \cdot 13^{2} \cdot 17}$ & $\pi^{42}$ & 1.156624 \\
\hline 5 & $\frac{2^{40}}{3^{20} \cdot 5^{8} \cdot 7^{6} \cdot 11 \cdot 13^{3} \cdot 17}$ & $\pi^{50}$ & 1.029466 \\
\hline 7 & $\frac{2^{40}}{3^{23} \cdot 5^{10} \cdot 7^{6} \cdot 11^{2} \cdot 13^{2} \cdot 17^{2}}$ & $\pi^{58}$ & 1.006025 \\
\hline 9 & $\frac{2^{21} \cdot 9413 \cdot 6782351}{3^{23} \cdot 5^{10} \cdot 7^{8} \cdot 11^{4} \cdot 13^{4} \cdot 17^{2} \cdot 19 \cdot 61}$ & $\pi^{66}$ & 1.000909 \\
\hline
\end{tabular}

\section{Acknowledgment}

The authors would like to thank the referee for her/his valuable comments and suggestions to improve the quality of the paper. This work was conducted as a part of the doctoral dissertation of the first author. We thank Professor Alexei Panchishkin for useful discussions.

\section{References}

[1] S. BÖCherer, "Über die Funktionalgleichung automorpher $L$-Funktionen zur Siegelschen Modulgruppe", J. Reine Angew. Math. 362 (1985), p. 146-168.

[2] F. Chiera \& K. VAnKov, "On special values of spinor L-functions of Siegel cusp eigenforms of genus 3", http://arxiv.org/abs/0805.2114, 2008. 
[3] M. Courtieu \& A. Panchishkin, Non-Archimedean L-functions and arithmetical Siegel modular forms, second ed., Lecture Notes in Mathematics, vol. 1471, Springer-Verlag, Berlin, 2004, viii+196 pages.

[4] P. Deligne, "Valeurs de fonctions $L$ et périodes d'intégrales", in Automorphic forms, representations and L-functions (Proc. Sympos. Pure Math., Oregon State Univ., Corvallis, Ore., 1977), Part 2, Proc. Sympos. Pure Math., XXXIII, Amer. Math. Soc., Providence, R.I., 1979, With an appendix by N. Koblitz and A. Ogus, p. 313-346.

[5] A. T. Do \& K. VAnKov, "Supplementary materials for the present article", http://jtnb. cedram.org/public/JTNB_2015_-27_3_727_0/DoVankov-suppl.pdf.

[6] B. H. Gross \& D. B. ZaGier, "Heegner points and derivatives of L-series", Invent. Math. 84 (1986), no. 2, p. 225-320.

[7] T. IKEDA, "Pullback of the lifting of elliptic cusp forms and Miyawaki's conjecture", Duke Math. J. 131 (2006), no. 3, p. 469-497.

[8] W. C. W. LI, "L-series of Rankin type and their functional equations", Math. Ann. 244 (1979), no. 2, p. 135-166.

[9] T. Miyake, Modular forms, english ed., Springer Monographs in Mathematics, SpringerVerlag, Berlin, 2006, Translated from the 1976 Japanese original by Yoshitaka Maeda, $\mathrm{x}+335$ pages.

[10] I. MiyaWAKI, "Numerical examples of Siegel cusp forms of degree 3 and their zeta-functions", Mem. Fac. Sci. Kyushu Univ. Ser. A 46 (1992), no. 2, p. 307-339.

[11] A. A. Panchishkin, "Two variable $p$-adic $L$ functions attached to eigenfamilies of positive slope", Invent. Math. 154 (2003), no. 3, p. 551-615.

[12] R. A. Rankin, "Contributions to the theory of Ramanujan's function $\tau(n)$ and similar arithmetical functions. I. The zeros of the function $\sum_{n=1}^{\infty} \tau(n) / n^{s}$ on the line $\Re s=13 / 2$. II. The order of the Fourier coefficients of integral modular forms", Proc. Cambridge Philos. Soc. 35 (1939), p. 351-372.

[13] J.-P. SERRE, "Formes modulaires et fonctions zêta p-adiques", in Modular functions of one variable, III (Proc. Internat. Summer School, Univ. Antwerp, 1972), Springer, Berlin, 1973, p. 191-268. Lecture Notes in Math., Vol. 350.

[14] G. Shimura, "The special values of the zeta functions associated with cusp forms", Comm. Pure Appl. Math. 29 (1976), no. 6, p. 783-804.

[15] — Elementary Dirichlet series and modular forms, Springer Monographs in Mathematics, Springer, New York, 2007, viii+147 pages.

[16] J. Sturm, "Projections of $C^{\infty}$ automorphic forms", Bull. Amer. Math. Soc. (N.S.) 2 (1980), no. 3, p. 435-439.

[17] D. ZAGIER, "Modular forms whose Fourier coefficients involve zeta-functions of quadratic fields", in Modular functions of one variable, VI (Proc. Second Internat. Conf., Univ. Bonn, Bonn, 1976), Springer, Berlin, 1977, p. 105-169. Lecture Notes in Math., Vol. 627.

Anh Tuan Do

Institut Fourier, Université Grenoble I,

38402 Saint Martin D'Hères

FRANCE

E-mail: anh-tuan.do@ujf-grenoble.fr

$U R L:$ http://www-fourier.ujf-grenoble.fr/

Kirill VANKOV

Institut Fourier, Université Grenoble I, 38402 Saint Martin D'Hères

FRANCE

E-mail: kirill.vankov@gmail.com 